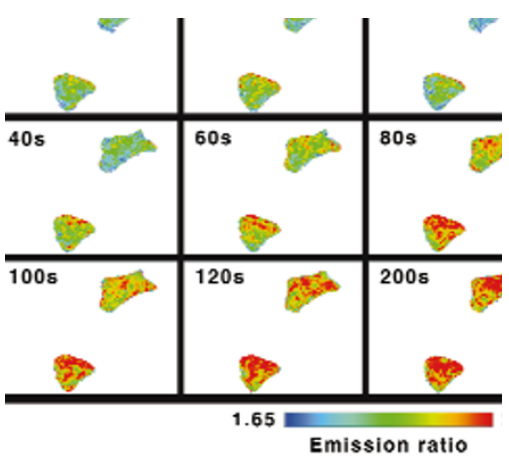

On page 313, Nagai et al. describe a way of visualizing phosphorylation activity of cAMP-dependent protein kinase (PKA) as it occurs in living cells. They placed a well-characterized substrate of PKA between blue and green mutants of GFP Phosphorylation of this peptide by PKA resulted in a conformational change that reduced fluorescence energy transfer (FRET) between the two GFPs. In COS-7 cells expressing the indicator, the reduced activity of PKA after treatment with kinase inhibitors could be monitored by FRET, suggesting that the fluorescent indicator might be useful in screens for kinase inhibitors.

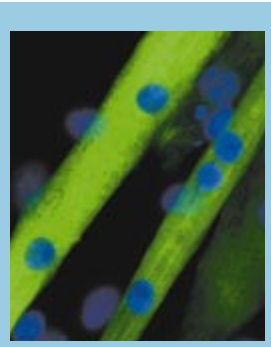

Limb regeneration in amphibians involves the disassembly of multinucleated myotubes into mononucleated fragments that grow and divide as single cells. To investigate whether myotube fragmentation could occur in mammals, Schultz and colleagues screened a combinatorial chemical library for compounds that could induce a similar change in morphology

cultured myotubes. One of the compounds identified was a microtubule-binding molecule, which

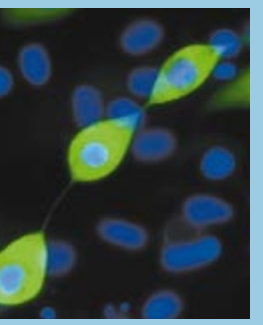
they called myoseverin. Interestingly, when myoseverin was removed from the medium, the cells underwent DNA synthesis and began to proliferate. Transcriptional profiling demonstrated that myoseverin treatment led to enhanced expression of a number of genes involved in wound repair, suggesting that the compound had primed the regenerative potential of the cells, and that it could find use in tissue engineering strategies.

Research Briefs written by Natalie DeWitt and Robert Frederickson.

\title{
Delivering PNAs to the nucleus
}

Peptide nucleic acids (PNAs) are synthetic homologs of nucleic

acids with the sugar-phosphate backbone replaced by an

uncharged mimic of repeating 2-aminoethyl-glycine units.

PNAs bind tightly to single-stranded complementary DNAs to

form PNA-DNA hybrids, and would be promising anti-gene

agents were it not for their limited ability to reach the cell nucle-

us. To address this problem, Boffa and colleagues examined

whether fusion of a PNA to a nuclear localization signal (NLS)

could potentiate their anti-gene effects. They show that an anti-myc PNA-NLS chimera was localized efficiently to the nucleus of cells derived from Burkitt's lymphoma that overexpress c-myc, and that expression of the latter was downregulated. These effects were associated with both inhibition of cell cycle progression and apoptosis (see pp. 303-303).

\section{Enantiomer selectivity inverted}

On page 317-320, May et al. use directed evolution to invert the enantioselectivity of hydantoinase, an enzyme used in the industrial production of L-methionine by Escherichia coli. Since hydantoinases are typically selective for the D- over the L-enantiomer, a significant amount of nonproductive intermediates accumulate, reducing the productivity for the L-amino acid. By introducing random mutations into the wild-type gene for hydantoinase, and then screening for gene products with altered enantioselectivity, the researchers converted a D-selective enzyme into an L-selective one. When whole E. coli cells expressed the evolved enzyme, it reduced levels of the unwanted intermediate and boosted productivity of the process.

\section{Sampling kinases in single cells}

Kinases and phosphatases play key roles in the diverse networks transmitting signals within and between cells. Most assays of physiological kinase activation involve measurements representing the average of the response of large populations of cells, which may or may not be heterogeneous in their intensity or time course. Moreover, the preparation of cellular extracts can often introduce inaccuracies into the process. Allbritton and colleagues have now developed a method to assay the activation of individual kinases within single mammalian cells. The method relies on chemical separation to identify and quantify fluorescent peptide substrates that have been injected into individual cells. The electrophoretic mobility of the substrates changes upon phosphorylation, and the latter are easily detected by capillary electrophoresis coupled to laser-induced fluorescence (see pp. 309-312).
Since plastids are not transmitted to pollen, expressing transgenes in chloroplast genomes (transplastomics) is viewed as a way of preventing the transgenes' spread to weedy relatives. Now on page 333 , Staub et al. show that human somatotropin, a therapeutic protein used to treat disorders such as hypopituitary dwarfism and HIV wasting syndrome, can be produced at high levels and in an active form in tobacco chloroplasts. Given that there are no known plastid-encoded proteins that contain disulfide bonds, it was particularly encouraging that the recombinant somatotropin was appropriately disulfide bonded.

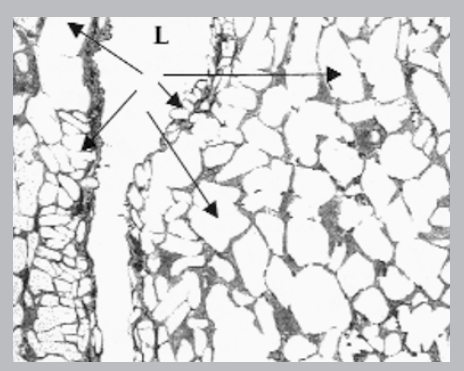

Between 1985 and 1992, thousands of cryopreserved veins were used for arterial bypass surgical procedures. The formation of extracellular ice during cryopreservation is known to be a hazard to structured organs and tissues, and conventional cryopreservation strategies have proved unsatisfactory. Vitrification is an alternate method of freezing that involves the solidification of an object in an amorphous glassy state that obviates the formation of ice crystals. In this issue, Taylor and colleagues have evaluated a vitrification approach to preserving vascular tissue, and show that it markedly improves the function of thawed tissues compared with those subjected to a standard crypreservation technique. The vitrified grafts were also shown to function in an autologous transplant model similarly to fresh tissue grafts (see pp. 296-299). 